\title{
PRESSÃO INTRA-ABDOMINAL NO TRANSPLANTE HEPÁTICO: SÉRIE PROSPECTIVA DE CASOS
}

\section{Intra-Abdominal Pressure in Hepatic Transplantation: Prospective Case Series}

\author{
Olival Cirilo Lucena da Fonseca Neto; Luiz Eduardo Rafael Moutinho; Ludmila Rodrigues de Oliveira Costa; \\ Priscylla Jennie Monteiro Rabêlo; Raimundo Hugo Matias Furtado; Fernando Jorge Diniz Cavalcanti; \\ Renata Ferreira Bezerra; Paulo Sergio Vieira de Melo; Américo Gusmão Amorim; Cláudio Moura Lacerda
}

\section{RESUMO}

Introdução: A hipertensão intra-abdominal (HIA) é uma complicação bastante frequente em pacientes críticos, que pode desencadear disfunção orgânica e mortalidade. O transplante de fígado produz diversos fatores de risco para a HIA e esse fenômeno pode subsidiar importantes informações sobre o desenvolvimento da lesão renal aguda (LRA) no pós-operatório precoce nesse grupo de doentes. Objetivo: Quantificar a pressão intra-abdominal (PIA), caracterizar a prevalência da HIA e avaliar a associação da HIA com a LRA nos receptores do transplante hepático. Métodos: Trata-se de um estudo observacional e analítico, realizado prospectivamente durante a pandemia do SARS-COV-2, composto por amostra de conveniência que incluiu pacientes submetidos ao transplante ortotópico de fígado, divididos em grupo $\mathrm{H}$ (PIA $>12 \mathrm{mmHg}$ ) e grupo $\mathrm{N}$ (PIA $<12 \mathrm{mmHg}$ ). Foi realizada uma recategorização para adequação do tamanho da nova amostra. Resultados: Foram realizados 16 transplantes de fígado, durante o período proposto do estudo, dos quais sete pacientes foram incluídos nesta análise. No total, 46 medidas da PIA foram executadas na amostra deste estudo. 0 índice de massa corporal, o Model for End-Stage Liver Disease Sodium (MELD-Na), o volume da ascite e a síndrome da reperfusão não distinguiram entre os grupos $\mathrm{H}$ e N. A PIA mediana foi de 10,5 mmHg, imediatamente após o transplante, com redução para $8.5 \mathrm{mmHg}$ decorridas 48 horas. O perfil hemodinâmico foi bastante semelhante entre os grupos, entretanto houve maior uso de drogas vasoativas no grupo $\mathrm{N}$ e de fluidos intravasculares no grupo $\mathrm{H}$. Conclusão: Não houve associação entre a LRA nos pacientes acometidos com HIA após o transplante hepático. Esse resultado pode não refletir com fidedignidade a relação da LRA e a HIA nessa população, devido ao poder da amostra dessa análise. Não foi possível estabelecer diferenças significativas entre os grupos $\mathrm{H}$ e N e a prevalência da HIA restringiu-se a níveis brandos. Os resultados sobre manejo hemodinâmico apoiam uma perspectiva mais otimista em relação ao uso de drogas vasoativas em detrimento ao uso irrestrito de fluidos intravasculares.

Descritores: Hipertensão Intra-abdominal, Transplante de Fígado, Lesão Renal Aguda.

Instituição:

Serviço de Cirurgia Geral e Transplante de Fígado do Hospital Universitário Oswaldo Cruz (HUOC) - Universidade de Pernambuco (UPE), Recife (PE), Brasil.

Correspondência:

Luiz Eduardo Rafael Moutinho

E-mail: luiz.moutinho@upe.br

https://doi.org/10.53855/bjt.v24i1.003

Recebido em 10/12/2020

\section{INTRODUÇÃO}

O abdome é um compartimento fechado, dotado de pressão regida pela interação entre o componente parietal e o conteúdo visceral. De acordo com World Society on Abdominal Compartment Syndrome (WSACS), os valores fisiológicos da pressão intraabdominal (PIA) variam de 2 a $7 \mathrm{mmHg}$ e a elevação sustentada acima de $12 \mathrm{mmHg}$ define o quadro de hipertensão intra-abdominal (HIA). ${ }^{1}$ 
A instalação do estado hipertensivo é causada por:

1) diminuição da complacência da parede abdominal,

2) aumento do conteúdo de lesões intra-abdominais,

3) aumento do conteúdo gastrointestinal e

4) aumento do extravasamento capilar.

A HIA é graduada segundo o perfil de pressão: grau I (12-15 $\mathrm{mmHg})$, grau II (16-20 $\mathrm{mmHg})$, grau III (21-25 $\mathrm{mmHg}$ ) e grau IV (>25 mmHg). ${ }^{2}$

A HIA tem uma importância clínica fundamental e é bastante frequente na Unidade de Terapia Intensiva (UTI): essa condição prevalece em $32-51 \%$ dos pacientes críticos nos maiores estudos prospectivos e foi associada, independentemente, ao aumento de mortalidade. $^{3,4} \mathrm{O}$ conjunto da fisiopatologia da HIA é mediado pelo desequilíbrio das pressões de perfusão e provoca disfunção múltipla de órgãos, com ameaça grave à vida do doente. Esse processo pode, criticamente, evoluir para síndrome compartimental abdominal, uma condição clínica definida pelo aumento sustentado da PIA acima de $20 \mathrm{mmHg}$ associado à nova disfunção orgânica.

A avaliação da PIA com base em parâmetros antropométricos e clínicos não é confiável. Medidas objetivas são recomendadas a todo paciente crítico com fator de risco para o desenvolvimento da HIA ${ }^{5} \mathrm{~A}$ PIA pode ser medida indiretamente através da bexiga urinária, um órgão extraperitoneal e intra-abdominal com paredes flexíveis que, quando plenamente infundido, pode refletir adequadamente a pressão interna do abdome. Trata-se de um método indireto, seguro e confiável. ${ }^{6}$

A lesão renal aguda (LRA) é uma complicação frequente no pós-transplante hepático: ela incide em $10-25 \%$ e determina um importante fator de mortalidade. ${ }^{7}$ Diversos mecanismos foram sugeridos e sabe-se hoje que a HIA é um fator de risco independente para a LRA nesse cenário. ${ }^{8,9}$

A HIA permanece como importante ponto no manejo intensivo no paciente submetido ao transplante de fígado e, portanto, este trabalho objetivou: quantificar os valores da PIA, caracterizar a prevalência da HIA e avaliar a associação dessa complicação quanto ao desenvolvimento de LRA no pós-operatório precoce do transplante.

\section{MATERIAIS E MÉTODOS}

\section{Desenho do Estudo}

Trata-se de um estudo observacional e analítico, realizado prospectivamente, composto por amostra de conveniência que incluiu pacientes submetidos ao transplante ortotópico de fígado e comparou-os em relação ao desenvolvimento de HIA (grupo $\mathrm{H}$ vs grupo $\mathrm{N}$ ), durante o período de janeiro de 2020 a agosto de 2020, na Unidade de Transplante de Fígado do Hospital Universitário Oswaldo Cruz (HUOC-PE).

\section{Critérios de Inclusão e Exclusão}

Pacientes submetidos ao transplante ortotópico de fígado pela técnica convencional sem desvio venovenoso e pela técnica Piggyback foram incluídos. Pacientes com idade menor que 18 anos; óbito no período transoperatório; transplante intervivos; transplante com hemitransposição porto-caval; transplante "split liver"; transplante duplo fígado/rim; retransplante e com hepatite fulminante foram excluídos. Pacientes com parada cardiorrespiratória transoperatória e com reanimação bem sucedida foram excluídos da análise da função renal e hepática precoce (Tabela 1), entretanto, excepcionalmente, incluídos na quantificação da PIA e caracterização da prevalência da HIA no pós-transplante (Tabela 2).

\section{Procedimentos e Definições}

A equipe de pesquisa acompanhou os pacientes durante o pré-operatório, no transcorrer cirúrgico e nas 48 horas pós-transplante, correspondente ao primeiro dia pósoperatório (1DPO) e ao segundo dia pós-operatório (2DPO). As variáveis do curso clínico e cirúrgico foram obtidas prospectivamente e registradas em protocolo de pesquisa desenvolvido para este trabalho. A equipe de pesquisa realizou sete medições da PIA em cada paciente incluído, a partir do pós-operatório imediato e a cada oito horas durante as 48 horas do internamento na UTI. A medida da PIA foi expressa em $\mathrm{mmHg}$, foi aferida na posição supina completa (decúbito dorsal), desde que na ausência de contratura abdominal, ao final da expiração, com um máximo de $25 \mathrm{ml}$ de solução salina instilada através de um cateter urinário. A leitura de pressão é zerada a nível axilar médio. ${ }^{1}$

A LRA foi avaliada conforme a creatinina sérica, segundo os critérios: I- Aumento de $1,5 \mathrm{vez}$ sobre o valor basal ou aumento absoluto de $0,3 \mathrm{mg} / \mathrm{dl}$ sobre o valor basal; II- Aumento de duas vezes sobre o valor basal; III- Aumento de três vezes sobre o valor basal ou valor absoluto maior que $4 \mathrm{mg} / \mathrm{dl}^{10}$

A disfunção primária do enxerto foi avaliada através da presença de, pelo menos, uma ou mais das seguintes anormalidades: bilirrubina maior ou igual a $10 \mathrm{mg} / \mathrm{dl}$, no $7^{\circ}$ dia pós-operatório, international normalized ratio (INR) maior ou igual a 1,6 no $7^{\circ}$ dia pós-operatório e alanina aminotransferase (ALT) ou aspartato aminotransferase (AST) maior que $2000 \mathrm{IU} / \mathrm{L}$ durante os sete primeiros dias. ${ }^{11}$ 
Olival Cirilo Lucena da Fonseca Neto; Luiz Eduardo Rafael Moutinho; Ludmila Rodrigues de Oliveira Costa; Priscylla Jennie Monteiro Rabêlo; Raimundo Hugo Matias Furtado; Fernando Jorge Diniz Cavalcanti; Renata Ferreira Bezerra; Paulo Sergio Vieira de Melo; Américo Gusmão Amorim; Claudio Moura Lacerda

Tabela 1: Função renal e função hepática precoce entre os pacientes com HIA ( $\geq 12 \mathrm{mmHg}$, grupo H) e sem (PIA $<12 \mathrm{mmHg}$, grupo $\mathrm{N}$ ) após o transplante de fígado.

\begin{tabular}{|c|c|c|c|c|}
\hline \multirow{2}{*}{ Variáveis } & Grupo N & Grupo H & Total & \multirow{2}{*}{ Valor de $p^{1}$} \\
\hline & $\%$ & $\%$ & $\%$ & \\
\hline \multicolumn{5}{|l|}{ Diurese $(\mathrm{ml})^{*}$} \\
\hline Operatório & $775(195 ; 1025)$ & $1450(800 ; 1500)$ & $912,5(195 ; 1500)$ & 0,200 \\
\hline $1 \mathrm{DPO}$ & $950(750 ; 1100)$ & 1025 (650; 1100) & $987,5(650 ; 1100)$ & 1,000 \\
\hline $2 \mathrm{DPO}$ & $1800(1250 ; 2150)$ & $780(575 ; 1650)$ & $1450(575 ; 2150)$ & 0,200 \\
\hline \multicolumn{5}{|l|}{ Creatinina* } \\
\hline Pré-operatório & $1,70(1,03 ; 2,06)$ & $1,20(0,70 ; 2,60)$ & $1,45(0,70 ; 2,60)$ & 1,000 \\
\hline $1 \mathrm{DPO}$ & $2,00(1,51 ; 2,68)$ & $1,80(0,60 ; 2,00)$ & $1,90(0,60 ; 2,68)$ & 0,400 \\
\hline $2 \mathrm{DPO}$ & $2,33(2,20 ; 2,81)$ & $2,48(1,30 ; 3,50)$ & $2,41(1,30 ; 3,50)$ & 1,000 \\
\hline \multicolumn{5}{|l|}{ LRA } \\
\hline Grau 0/I & 66,7 & 33,3 & 50,0 & \\
\hline Grau II/III & 33,3 & 66,7 & 50,0 & $1,000^{2}$ \\
\hline \multicolumn{5}{|l|}{ INR* } \\
\hline Pré-operatório & $1,80(1,24 ; 6,58)$ & $2,79(1,60 ; 2,92)$ & $2,30(1,24 ; 6,58)$ & 1,000 \\
\hline $1 \mathrm{DPO}$ & $3,22(2,07 ; 4,05)$ & $3,15(2,15 ; 3,30)$ & $3,19(2,07 ; 4,05)$ & 1,000 \\
\hline $2 \mathrm{DPO}$ & $2,18(1,75 ; 2,58)$ & $2,55(1,13 ; 2,90)$ & $2,37(1,13 ; 2,90)$ & 1,000 \\
\hline \multicolumn{5}{|l|}{ AST* } \\
\hline Pré-operatório & $317,5(290 ; 345)$ & $112(37 ; 244)$ & $244(37 ; 345)$ & 0,200 \\
\hline $1 \mathrm{DPO}$ & $1187(366 ; 1912)$ & $439(426 ; 1202)$ & $813(366 ; 1912)$ & 1,000 \\
\hline $2 \mathrm{DPO}$ & $977(177 ;$ 1155) & $399(199 ; 938)$ & $668,5(177 ; 1155)$ & 0,700 \\
\hline \multicolumn{5}{|l|}{$\mathrm{ALT}^{*}$} \\
\hline Pré-operatório & $169(131 ; 207)$ & $66(22 ; 178)$ & $131(22 ; 207)$ & 0,400 \\
\hline $1 \mathrm{DPO}$ & $1107(208 ; 2140)$ & $292(141 ; 866)$ & $579(141 ; 2140)$ & 0,400 \\
\hline $2 \mathrm{DPO}$ & $827(119 ; 1536)$ & $224(138 ; 652)$ & $438(119 ; 1536)$ & 0,700 \\
\hline \multicolumn{5}{|l|}{ BT* } \\
\hline Pré-operatório & $11,5(5,5 ; 33,5)$ & $5,5(2,0 ; 8,9)$ & $7,2(2,0 ; 33,5)$ & 0,400 \\
\hline $1 \mathrm{DPO}$ & $4,6(4,0 ; 16,3)$ & $3,2(1,5 ; 6,4)$ & $4,3(1,5 ; 16,3)$ & 0,400 \\
\hline $2 \mathrm{DPO}$ & $3,2(3,0 ; 11,7)$ & $3,5(1,2 ; 5,9)$ & $3,2(1,2 ; 11,7)$ & 0,800 \\
\hline \multicolumn{5}{|l|}{ DE } \\
\hline Não & 100,0 & 100,0 & 100,0 & - \\
\hline
\end{tabular}

* Resultados apresentados sob a forma de mediana (mín; máx)

${ }^{I}$ Teste de Mann Whitney

${ }^{2}$ Teste exato de Fisher 
Pressão intra-abdominal no transplante hepático: série prospectiva de casos

Tabela 2: Distinção das técnicas operatórias do transplante hepático e manutenção hemodinâmica do receptor transoperatória

\begin{tabular}{|c|c|c|c|c|}
\hline \multirow{2}{*}{$\begin{array}{c}\text { Dados } \\
\text { Transoperatórios }\end{array}$} & Grupo N & Grupo H & Total & \multirow{2}{*}{ Valor de $\mathbf{p}^{1}$} \\
\hline & $\%$ & $\%$ & $\%$ & \\
\hline \multicolumn{5}{|l|}{ Tranplante } \\
\hline Convencional & 100,0 & 66,7 & 85,7 & \multirow{2}{*}{$0,429^{2}$} \\
\hline Piggyback & 0,0 & 33,3 & 14,3 & \\
\hline \multicolumn{5}{|l|}{ Anastomose Biliar } \\
\hline Biliodigestiva & 25,0 & 66,7 & 42,9 & \multirow{2}{*}{$0,486^{2}$} \\
\hline Colédoco-colédoco & 75,0 & 33,3 & 57,1 & \\
\hline \multicolumn{5}{|l|}{ Sind Reperfusão } \\
\hline Não & 50,0 & 66,7 & 57,1 & \multirow{2}{*}{$1,000^{2}$} \\
\hline Sim & 50,0 & 33,3 & 42,9 & \\
\hline \multicolumn{5}{|l|}{ Tempo (minutos)* } \\
\hline Cirurgia & $336,5(310 ; 375)$ & $297(295 ; 575)$ & $330(295 ; 575)$ & 0,629 \\
\hline Anestesia & $417,5(380 ; 465)$ & $370(360 ; 690)$ & $415(360 ; 690)$ & 0,629 \\
\hline Isquemia fria & $363,5(260 ; 478)$ & $235(226 ; 714)$ & $263(226 ; 714)$ & 0,629 \\
\hline Isquemia quente & $32(26 ; 45)$ & $35(23 ; 41)$ & $32(23 ; 45)$ & 1,000 \\
\hline \multicolumn{5}{|l|}{ Reposição* } \\
\hline Cristaloide (ml) & $3850(2500 ; 4500)$ & $5000(3500 ; 7000)$ & $4000(2500 ; 7000)$ & 0,400 \\
\hline Coloide (ml) & $275(0 ; 400)$ & $700(500 ; 1150)$ & $400(0 ; 1150)$ & 0,057 \\
\hline Hemácias & $2(0 ; 9)$ & $6(0 ; 7)$ & $4(0 ; 9)$ & 0,857 \\
\hline Plasma & $2(0 ; 4)$ & $4(0 ; 6)$ & $4(0 ; 6)$ & 0,629 \\
\hline \multicolumn{5}{|l|}{ Pesos (g)* } \\
\hline Explante & $3101(1772 ; 3368)$ & $1260(1040 ; 2644)$ & $2208(1040 ; 3368)$ & 0,200 \\
\hline Enxerto & $1588(1180 ; 2152)$ & $1250(800 ; 1450)$ & $1306(800 ; 2152)$ & 0,400 \\
\hline Razão (\%) & $60,3 \%(38,8 ; 121,4)$ & $63,5 \%(47,3 ; 139,4)$ & $61,9 \%(38,8 ; 139,4)$ & 0,700 \\
\hline \multicolumn{5}{|l|}{ Final da cirurgia* } \\
\hline PAM $(\mathrm{mmHg})$ & $75(60 ; 80)$ & $78(57 ; 99)$ & $78(57 ; 99)$ & 1,000 \\
\hline $\mathrm{PVC}(\mathrm{mmHg})$ & $10(10 ; 12)$ & $10,5(10 ; 11)$ & $10(10 ; 12)$ & 1,000 \\
\hline DVA $(\mathrm{ml} / \mathrm{h})$ & $26(10 ; 60)$ & $6(5 ; 80)$ & $15(5 ; 80)$ & 0,629 \\
\hline
\end{tabular}

* Resultados apresentados sob a forma de mediana (mín; máx)

${ }^{1}$ Teste de Mann Whitney

${ }^{2}$ Teste exato de Fisher 
Olival Cirilo Lucena da Fonseca Neto; Luiz Eduardo Rafael Moutinho; Ludmila Rodrigues de Oliveira Costa; Priscylla Jennie Monteiro Rabêlo; Raimundo Hugo Matias Furtado; Fernando Jorge Diniz Cavalcanti; Renata Ferreira Bezerra; Paulo Sergio Vieira de Melo;

Américo Gusmão Amorim; Claudio Moura Lacerda

A síndrome pós-reperfusão é uma complicação transoperatória que envolve distúrbios cardiovasculares e metabólicos dramáticos, após a reperfusão do enxerto. Ela foi definida pela diminuição de $30 \%$ na pressão arterial média até cinco minutos do início da reperfusão e com duração maior que um minuto. ${ }^{12}$

\section{Análise dos Dados}

A análise dos dados e o processamento dos grupos para análise bivariada foram planejados segundo dois critérios distintos e não exclusivos: perfil de PIA (H e N) e técnica cirúrgica (TC e TP). Categorização inicial:

Grupo H- Pacientes transplantados que apresentarem graus 3 e 4 de HIA.

Grupo N- Pacientes transplantados que apresentarem graus 1 e 2 de HIA ou níveis normais de PIA.

Grupo TC- Pacientes transplantados com o uso da técnica Convencional sem desvio veno-venoso.

Grupo TP- Pacientes transplantados com o uso da técnica Piggyback.

Foi realizada uma recategorização devido à adequação da nova amostra. A divisão com base nas técnicas cirúrgicas foi excluída da análise. O agrupamento com base nos níveis de PIA foi fundamentado para uma análise bivariada e o reenquadramento foi realizado conforme exposto a seguir.

Recategorização:
Grupo H- Pacientes transplantados que apresentaram graus 1 ou maior de HIA ( $\geq 12 \mathrm{mmHg}$ ).

Grupo N- Pacientes transplantados que não apresentaram HIA (<12 mmHg).

A fim de comparar os grupos de pacientes diagnosticados com e sem HIA, foi utilizado o teste exato de Fisher, com respeito às variáveis de interesse categóricas/ qualitativas, sendo obtidas as frequências absoluta $(\mathrm{N})$ e relativa (\%) dessas variáveis. O teste de Mann-Whitney foi aplicado no caso de variáveis quantitativas, estas apresentadas sob a forma de mediana (mínimo; máximo). A PIA foi aferida em sete momentos pós-cirúrgicos e para descrevê-las numericamente foram utilizadas medidas de posição, tais como média e mediana, e medida de dispersão, o desvio-padrão. Foram construídos Box-Plots com o intuito de comparar essas aferições graficamente, ao longo do tempo (Figura 1).

As conclusões foram tomadas ao nível de $5 \%$ de significância.

\section{Aspectos Éticos}

O parecer consubstanciado do Conselho de Ética e Pesquisa do Complexo Hospitalar HOUC/PROCAPE do projeto foi aprovado em conformidade com as Resoluções 466/12, do CNS-MS não havendo, portanto, nenhum impedimento ético à sua realização. O número do parecer e do CAAE do projeto, submetidos através da Plataforma Brasil, são, respectivamente, 3.269.530 e 96218318.5.0000.5192.

Figura 1: Box plot: comportamento da Pressão Intra-Abdominal (PIA) no pós-operatório precoce do transplante de fígado

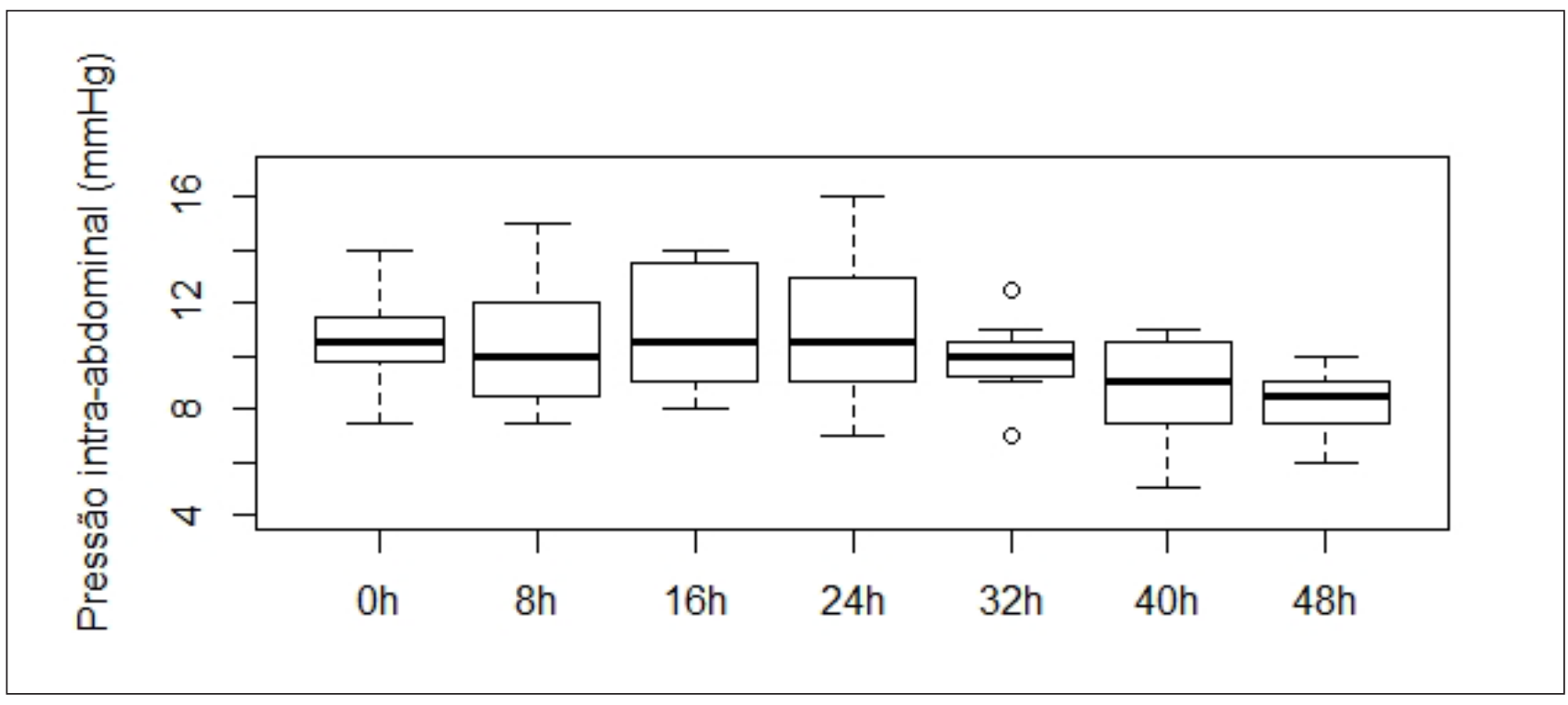




\section{RESULTADOS}

Foram realizados 16 transplantes de fígado no centro durante o período proposto, dos quais $\mathrm{N}=7$ pacientes foram incluídos e 46 medidas de PIA foram realizadas. A taxa de recrutamento e obtenção completa dos dados foi $44 \%$. Nenhum paciente se recusou a participar da pesquisa. $\mathrm{O}$ insucesso de inclusão da amostra em $77,8 \%$ dos casos excluídos ocorreu devido à logística do procedimento de coleta e, portanto, incompletude dos dados.

A população da amostra foi predominantemente masculina, acometida com cirrose hepática secundária ao álcool. O IMC, o MELD-Na e a ascite não distinguiram entre os grupos $\mathrm{HeN}$ (Tabela 1). A síndrome da reperfusão esteve presente de forma semelhante em ambos os grupos. Não houve diferença dos tempos cirúrgico e anestésico, assim como dos tempos de isquemia. O peso do enxerto em razão do fígado explantado não foi fator de risco para HIA; entretanto, na amostra não houve qualquer transplante com enxerto mais pesado que $4 \%$ do peso corporal do receptor (Tabela 2).
O perfil hemodinâmico foi bastante semelhante entre os grupos, todavia há uma mediana maior para o uso de drogas vasoativas no grupo que não desenvolveu HIA (Tabela 2).

Os dados deste trabalho expõem PIA de $10,5 \mathrm{mmHg}$, imediatamente após o transplante, com redução para 8.5 $\mathrm{mmHg}$ após 48 horas, sem diferença estatisticamente significante (Figura 1). Nenhum paciente foi acometido gravemente pela HIA (graus III e IV) e a amostra restringiu-se a pacientes com aumentos brandos da PIA. Entretanto, $75 \%$ dos pacientes apresentaram, em algum momento do pós-operatório, HIA (PIA maior ou igual a $12 \mathrm{mmHg}$ ).

Os dados de um paciente foram excluídos, especificamente, da análise sobre a LRA, conforme o critério estabelecido na metodologia de parada cardiorrespiratória transoperatória com reanimação bem sucedida.

Tabela 3 - Características dos pacientes com Hipertensão Intra-Abdominal (HIA) ( $\geq 12$ mmHg, grupo H) e sem (PIA <12 mmHg, grupo N).

\begin{tabular}{|c|c|c|c|c|c|c|c|}
\hline \multirow{2}{*}{ Dados Demográficos } & \multicolumn{2}{|c|}{ Grupo N } & \multicolumn{2}{|c|}{ Grupo H } & \multicolumn{2}{|c|}{ Total } & \multirow{2}{*}{$\begin{array}{c}\text { Valor de } \\
\mathbf{p}^{1}\end{array}$} \\
\hline & $\mathbf{N}$ & $\%$ & $\mathbf{N}$ & $\%$ & $\mathbf{N}$ & $\%$ & \\
\hline \multicolumn{8}{|l|}{ Sexo } \\
\hline Masculino & 3 & 75,0 & 2 & 66,7 & 5 & 71,4 & \\
\hline Feminino & 1 & 25,0 & 1 & 33,3 & 2 & 28,6 & $1,000^{2}$ \\
\hline Idade (anos)* & \multicolumn{2}{|c|}{$41(27 ; 62)$} & \multicolumn{2}{|c|}{$46(21 ; 62)$} & \multicolumn{2}{|c|}{$46(21 ; 62)$} & 0,857 \\
\hline Peso (Kg)* & \multicolumn{2}{|c|}{$74,5(61 ; 120)$} & \multicolumn{2}{|c|}{$80(70 ; 104)$} & \multicolumn{2}{|c|}{$80(61 ; 120)$} & 0,857 \\
\hline Altura $(\mathrm{m})^{*}$ & \multicolumn{2}{|c|}{$1,65(1,58 ; 1,75)$} & \multicolumn{2}{|c|}{$1,73(1,64 ; 1,76)$} & \multicolumn{2}{|c|}{$1,65(1,58 ; 1,76)$} & 0,400 \\
\hline IMC* & \multicolumn{2}{|c|}{$25,71(24,44 ; 44,62)$} & \multicolumn{2}{|c|}{$26,73(26,03 ; 33,57)$} & \multicolumn{2}{|c|}{$26,45(24,44 ; 44,62)$} & 0,629 \\
\hline Ascite $(\mathrm{ml})^{*}$ & \multicolumn{2}{|c|}{$1000(500 ; 5000)$} & \multicolumn{2}{|c|}{$2000(750 ; 7000)$} & \multicolumn{2}{|c|}{$1000(500 ; 7000)$} & 0,629 \\
\hline MELD-Na* & \multicolumn{2}{|c|}{$29(18 ; 45)$} & \multicolumn{2}{|c|}{$23(22 ; 35)$} & \multicolumn{2}{|c|}{$25(18 ; 45)$} & 0,857 \\
\hline \multicolumn{8}{|l|}{ Diagnóstico } \\
\hline Cirrose por álcool & 1 & 25,0 & 2 & 66,7 & 3 & 42,9 & \\
\hline Cirrose por NASH & 1 & 25,0 & 0 & 0,0 & 1 & 14,3 & \\
\hline CEP & 1 & 25,0 & 0 & 0,0 & 1 & 14,3 & \\
\hline Sind Budd-Chiari & 1 & 25,0 & 1 & 33,3 & 2 & 28,6 & $1,000^{2}$ \\
\hline
\end{tabular}

* Resultados apresentados sob a forma de mediana (mín; máx)

${ }^{1}$ Teste de Mann Whitney

${ }^{2}$ Teste exato de Fisher 
Olival Cirilo Lucena da Fonseca Neto; Luiz Eduardo Rafael Moutinho; Ludmila Rodrigues de Oliveira Costa; Priscylla Jennie Monteiro Rabêlo; Raimundo Hugo Matias Furtado; Fernando Jorge Diniz Cavalcanti; Renata Ferreira Bezerra; Paulo Sergio Vieira de Melo; Américo Gusmão Amorim; Claudio Moura Lacerda

\section{DISCUSSÃO}

A alteração dramática do conteúdo abdominal após o transplante hepático pode resultar em alterações importantes da PIA, dado que existe um continente limitado dentro do abdome. O sangramento intraabdominal causado tanto pela manipulação cirúrgica quanto pela coagulopatia, o edema intestinal causado pelo clampeamento da veia porta durante a fase anepática, o extravasamento capilar causado pela reposição de volume e o acúmulo de líquido ascítico podem ocorrer no pós-operatório precoce e determinar fatores importantes para instalação da HIA.

Em uma análise com 62 pacientes submetidos ao transplante de fígado, $38,7 \%$ desenvolveram HIA e, no terceiro dia pós-operatório, todos tiveram regressão do quadro. Todavia, o critério para HIA utilizado neste outro estudo foi mais rigoroso e considerou apenas pacientes com PIA maior ou igual a $20 \mathrm{mmHg} .{ }^{9} \mathrm{O}$ presente estudo não constatou nenhum paciente com mesma magnitude de acometimento.

Discute-se, portanto, qual o limiar de PIA que pode desencadear, de fato, repercussões hemodinâmicas importantes ao doente. Biancofiori et al ${ }^{8}$ demonstrou que o valor de $25 \mathrm{mmHg}$ apresenta melhor sensibilidade e especificidade através da curva característica de operação (ROC curve) para insuficiência renal (creatinina sérica absoluta maior que $1,5 \mathrm{mg} / \mathrm{dl}$ ou aumento relativo de $1,1 \mathrm{mg} / \mathrm{dl}$ ) no transplante hepático precoce. Entretanto, em pacientes graves na UTI a HIA foi um fator preditivo independente de LRA, mesmo em níveis de pressão tão baixos quanto $12 \mathrm{mmHg} .13$

Os resultados deste estudo não foram capazes de associar a HIA e a LRA (Tabela 3). Vale ressaltar que a amostra deste estudo compreendeu pacientes sem aumentos críticos de PIA. Em 2003, o primeiro grande estudo prospectivo sobre PIA com pacientes submetidos ao transplante hepático constatou que o desenvolvimento de LRA foi acompanhado com PIA mediana de $27 \mathrm{mmHg}$, enquanto o grupo isento dessa complicação demonstrou valores menores (mediana de $19 \mathrm{mmHg}$ ), com diferença estatisticamente significante..$^{14} \mathrm{Em} \mathrm{2007}$, outro grande estudo com pacientes transplantados também observou uma prevalência significativamente maior de LRA no grupo que desenvolveu $\mathrm{HIA}$, comparado àquele de PIA normal (46\% vs $8 \%) .{ }^{9}$ A HIA é associada ao aumento da pressão intraglomerular, ao desvio de sangue do córtex para a medula renal, à elevação da pressão venosa renal e à modulação das vias do balanço hídrico..$^{15} \mathrm{De}$ fato, diversas condições que envolvem o procedimento de transplante de fígado podem afetar o funcionamento renal precoce; no entanto, a HIA é fator independente para LRA nesse grupo de doentes.

As alterações fisiopatológicas da HIA afetam direta e indiretamente diversos sistemas do corpo, sendo reportadas, principalmente. alterações no sistema cardiovascular, respiratório, renal, hepatoesplênico e nervoso central. O quadro assemelha-se a uma lesão por isquemia-reperfusão, conferindo à HIA um mecanismo de má perfusão orgânica. ${ }^{16}$

A restauração da volemia e perfusão tecidual do paciente é essencial e deve ser realizado com a combinação tanto de fluidos quanto de vasoativos. Nota-se que o volume reposto no transoperatório no grupo $\mathrm{H}$ foi maior comparado ao grupo $\mathrm{N}$, entretanto sem diferença estatística. De fato, trata-se de um grupo de doentes submetidos a uma reposição importante de fluidos: os pacientes da amostra foram submetidos a uma soma mediana de $4000 \mathrm{ml}$ de cristaloides, 400 $\mathrm{ml}$ de coloides, quatro unidades de concentrado de hemácias e quatro unidades de plasma fresco durante o transplante (Tabela 2).

Ressalta-se que o uso excessivo de cristaloides é um fator desencadeante da HIA e, até mesmo, síndrome compartimental abdominal secundária. $\mathrm{O}$ uso irrestrito de volume intravascular é, desse modo, bastante reconhecido no comprometimento da sobrevida do doente. A manutenção da pressão de perfusão abdominal (PAM subtraído pela PIA) é um parâmetro importante para adequar a terapia de fluidos, demonstrando benefícios em razão de prevenir a HIA.17

Um dos pacientes não realizou fechamento primário da fáscia abdominal devido a edema intestinal e permaneceu com abertura temporária por mais de 48 horas. A PIA foi monitorada durante o acompanhamento pós-operatório e não houve HIA. A manutenção do abdômen aberto com fechamento temporário pode ser uma alternativa factível frente à síndrome compartimental abdominal recorrente em diversos contextos. ${ }^{18} \mathrm{O}$ paciente permaneceu na amostra da pesquisa.

A parada cardiorrespiratória com utilização de técnicas mecânicas e farmacológicas de reanimação ocorre em $5,5 \%$ dos transplantes de fígado e prevalecem após a revascularização hepática. Esse critério específico de exclusão foi utilizado porque a interrupção do débito cardíaco e as estratégias farmacológicas preconizadas durante a parada cardiorrespiratória preterem o fluxo sanguíneo renal e predispõem, naturalmente, à LRA. Entretanto, não ocorre alteração da PIA concomitante e esse fenômeno pode caracterizar um confundidor para a amostra analisada neste trabalho. ${ }^{19}$ 
O estudo tem um limite de impressão de respostas significantes devido à amostra reduzida. $O$ desenho do estudo é observacional e a prevalência da HIA é distinta dos demais estudos pelo desenvolvimento crescente do manejo do paciente com HIA ao longo das últimas duas décadas. Os pacientes foram submetidos ao tratamento preconizado da UTI e, possivelmente, foram aplicadas medidas conflitantes com o aumento da PIA.

A limitação da amostra justifica-se em especial por alguns pontos excepcionais. O número absoluto de transplantes de fígado realizados, durante a coleta de dados, foi bastante inferior ao estimado posteriormente, assim como a taxa de recrutamento e obtenção completa dos dados foi superestimada. Deve-se esse prejuízo à carência de uma equipe de pesquisa maior, ao isolamento social e à restrição à UTI previstos pela pandemia do SARS-COV-2.

\section{CONCLUSÃO}

Não houve associação entre a LRA nos pacientes acometidos com HIA após o transplante hepático. Esse resultado pode não refletir com fidedignidade a relação da LRA e a HIA nessa população. Não foi possível estabelecer diferenças significativas entre os grupos $\mathrm{He}$ $\mathrm{N}$, e a prevalência de HIA restringiu-se a níveis brandos. Os resultados sobre manejo hemodinâmico, apesar de sem significância estatística, apoiam uma perspectiva mais otimista em relação ao uso de drogas vasoativas em detrimento do uso maciço de fluidos.

\section{ABSTRACT}

Introduction: Intra-abdominal hypertension (IAH) is a common complication in critically ill patients, which can trigger organ dysfunction and mortality. Liver transplant can produce several risk factors related to IAH, and such phenomenon may subsidize important information on the development of acute kidney injury in the early postoperative period in this group of patients. Purpose: This article aims to quantify intra-abdominal pressure (IAP), describe IAH prevalence and assess the association of IAH and AKI in liver transplanted recipients. Methods: This is an observational and analytical study, prospectively carried out during the SARS-COV-2 pandemic. It was composed by a convenience sample that included patients submitted to orthotopic liver transplantation divided into groups $\mathrm{H}$ (IAP $>12 \mathrm{mmHg}$ ) and $\mathrm{N}$ (IAP $<12 \mathrm{mmHg}$ ). A re-categorization was performed to adapt the new sample size. Results: 16 liver transplants were performed during the period of the study, from which seven patients were included in these analyses. A total of 46 IAP measurements were carried out on the sample. There was no difference between groups $\mathrm{H}$ and $\mathrm{N}$ in Body Mass Index (BMI), Model for End-Stage Liver Disease Sodium (MELD-Na), ascites volume and reperfusion syndrome. The median IAP was $10.5 \mathrm{mmHg}$ immediately after transplantation, which was reduced to $8.5 \mathrm{mmHg}$ after 48 hours. The hemodynamic profile was quite similar between groups. However, there was a greater use of vasoactive drugs in group $\mathrm{N}$ and intravascular fluids in group $\mathrm{H}$. Conclusion: There was no association between AKI in patients affected by IAH after liver transplantation. This result may not reflect the relationship between AKI and IAH in this population due to the size of the studied sample. It was not possible to establish significant differences between groups $\mathrm{H}$ and $\mathrm{N}$, and the prevalence of IAH was restricted to moderate levels. The results of hemodynamic management suggest that better outcomes may be obtained by using vasoactive drugs instead of large volumes of intravascular fluids.

Keywords: Intra-abdominal Hypertension, Liver Transplantation, Acute Kidney Injury. 
Olival Cirilo Lucena da Fonseca Neto; Luiz Eduardo Rafael Moutinho; Ludmila Rodrigues de Oliveira Costa; Priscylla Jennie Monteiro Rabêlo; Raimundo Hugo Matias Furtado; Fernando Jorge Diniz Cavalcanti; Renata Ferreira Bezerra; Paulo Sergio Vieira de Melo;

Américo Gusmão Amorim; Claudio Moura Lacerda

\section{REFERÊNCIAS}

1. Kirkpatrick AW, Roberts DJ, De Waele J, Jaeschke R, Malbrain MLNG, De Keulenaer B, et al. Intra-abdominal hypertension and the abdominal compartment syndrome: updated consensus definitions and clinical practice guidelines from the World Society of the Abdominal Compartment Syndrome. Intensive Care Med. julho de 2013;39(7):1190-206.

2. Papavramidis TS, Marinis AD, Pliakos I, Kesisoglou I, Papavramidou N. Abdominal compartment syndromeIntra-abdominal hypertension: Defining, diagnosing, and managing. J Emerg Trauma Shock [Internet]. 2011 [citado 24 de outubro de 2020];4(2):279-91. Disponível em: https:// www.ncbi.nlm.nih.gov/pmc/articles/PMC3132369/

3. Kyoung K-H, Hong S-K. The duration of intra-abdominal hypertension strongly predicts outcomes for the critically ill surgical patients: a prospective observational study. World J Emerg Surg WJES. 2015;10:22.

4. Malbrain MLNG, Chiumello D, Pelosi P, Bihari D, Innes R, Ranieri VM, et al. Incidence and prognosis of intraabdominal hypertension in a mixed population of critically ill patients: a multiple-center epidemiological study. Crit Care Med. fevereiro de 2005;33(2):315-22.

5. Sugrue M, Bauman A, Jones F, Bishop G, Flabouris A, Parr $\mathrm{M}$, et al. Clinical examination is an inaccurate predictor of intraabdominal pressure. World J Surg. dezembro de 2002;26(12):1428-31.

6. Kron IL, Harman PK, Nolan SP. The measurement of intra-abdominal pressure as a criterion for abdominal reexploration. Ann Surg. janeiro de 1984;199(1):28-30.

7. Gainza FJ, Valdivieso A, Quintanilla N, Errazti G, Gastaca M, Campo M, et al. Evaluation of acute renal failure in the liver transplantation perioperative period: incidence and impact. Transplant Proc. fevereiro de 2002;34(1):250-1.

8. Biancofiore G, Bindi L, Romanelli AM, Bisà M, Boldrini A, Consani $G$, et al. Renal failure and abdominal hypertension after liver transplantation: determination of critical intraabdominal pressure. Liver Transplant Off Publ Am Assoc Study Liver Dis Int Liver Transplant Soc. dezembro de 2002;8(12):1175-81.

9. Shu M, Peng C, Chen H, Shen B, Zhou G, Shen C, et al. Intra-abdominal hypertension is an independent cause of acute renal failure after orthotopic liver transplantation. Front Med China. maio de 2007;1(2):167-72.

10. Mehta RL, Kellum JA, Shah SV, Molitoris BA, Ronco C, Warnock DG, et al. Acute Kidney Injury Network: report of an initiative to improve outcomes in acute kidney injury. Crit Care Lond Engl. 2007;11(2):R31.
11. Olthoff KM, Kulik L, Samstein B, Kaminski M, Abecassis M, Emond J, et al. Validation of a current definition of early allograft dysfunction in liver transplant recipients and analysis of risk factors. Liver Transplant Off Publ Am Assoc Study Liver Dis Int Liver Transplant Soc. 2010 ago;16(8):943-9.

12. Siniscalchi A, Gamberini L, Laici C, Bardi T, Ercolani $G$, Lorenzini L, et al. Post reperfusion syndrome during liver transplantation: From pathophysiology to therapy and preventive strategies. World $\mathrm{J}$ Gastroenterol [Internet]. 28 de janeiro de 2016 [citado 27 de outubro de 2020];22(4):1551-69. Disponível em: https://www.ncbi.nlm.nih.gov/pmc/articles/ PMC4721988/

13. Dalfino L, Tullo L, Donadio I, Malcangi V, Brienza N. Intra-abdominal hypertension and acute renal failure in critically ill patients. Intensive Care Med. abril de 2008;34(4):707-13.

14. Biancofiore G, Bindi ML, Romanelli AM, Boldrini A, Consani G, Bisà M, et al. Intra-abdominal pressure monitoring in liver transplant recipients: a prospective study. Intensive Care Med. janeiro de 2003;29(1):30_ 6.

15. Doty JM, Saggi BH, Blocher CR, Fakhry I, Gehr T, Sica D, et al. Effects of increased renal parenchymal pressure on renal function. J Trauma. maio de 2000;48(5):874-7.

16. Malbrain MLNG. Is it wise not to think about intraabdominal hypertension in the ICU? Curr Opin Crit Care. abril de 2004;10(2):132-45.

17. Cheatham ML, White MW, Sagraves SG, Johnson JL, Block EF. Abdominal perfusion pressure: a superior parameter in the assessment of intra-abdominal hypertension. J Trauma. outubro de 2000;49(4):621-6; discussion 626-627.

18. Hobeika C, Allard M-A, Bucur P-O, Naili S, Sa Cunha A, Cherqui D, et al. Management of the Open Abdomen after Liver Transplantation. World J Surg [Internet]. 1o de dezembro de 2017 [citado 26 de junho de 2020];41(12):3199-204. Disponível em: https://doi. org/10.1007/s00268-017-4125-1

19. Matsusaki T, Hilmi IA, Planinsic RM, Humar A, Sakai T. Cardiac arrest during adult liver transplantation: A single institution's experience with 1238 deceased donor transplants. Liver Transpl [Internet]. 2013 [citado 29 de outubro de 2020];19(11):1262-71. Disponível em: https://aasldpubs.onlinelibrary.wiley.com/doi/ abs/10.1002/lt.23723 